\title{
Medial hypothalamic stimulation decreases the phagocytic activity of the reticuloendothelial system
}

\author{
PAUL L. LAMBERT and ERNEST H. HARRELL \\ North Texas State University, Denton, Texas 76203 \\ and \\ JEAN ACHTERBERG \\ University of Texas Health Science Center, Dallas, Texas 75235
}

\begin{abstract}
Although research has linked the central nervous system with changes in immunoresponsivity, research on the possible role of the central nervous system in altering reticuloendothelial activity is lacking. This study investigated the possible relationship between hypothalamic structures and changes in responsivity of the reticuloendothelial system. Eight male albino rats received bilateral electrode implants in the ventromedial area of the hypothalamus, and, following brain stimulation, reticuloendothelial activity was assessed $3,6,12,24$, and $96 \mathrm{~h}$ after stimulation. Brain stimulation decreased phagocytic activity of the reticuloendothelial system. These findings may increase our understanding of a possible neural mechanism underlying relationships between stress and resistance to disease states.
\end{abstract}

Recently, several investigations have established a relationship between the hypothalamus and functioning of the immune system. For instance, lesions of hypothalamic structures have prevented antibody formation, tumor growth, and anaphylactic shock (Kavetsky, Turkevich, Akimova, Khayetsky, \& Matuechuck, 1969; Macris, Schiavi, Camerino, \& Stein, 1975; Schiavi, Adams, \& Stein, 1966), and stimulation of hypothalamic areas has been shown to increase antibody levels (Fessel \& Forsyth, 1963; Korneva \& Khai, 1963). Also, recordings of neuronal activity increased in medial hypothalamic areas simultaneously with IgM antibody synthesis in the spleen (Besedovsky \& Sorkin, 1977).

In attempting to determine the role of hypothalamic mechanisms in immunity, investigators have consistently overlooked a major component of the immune response known as the reticuloendothelial system (RES). Research has demonstrated that the RES removes toxins, injured cells, bacteria, and other foreign substances from the bloodstream by the process of phagocytosis (Carr, 1973). In addition to phagocytosis, macrophages have been shown to interact with other components of the immune system (Friedman, 1960; Frobisher, Hindsill, Crabtree, \& Goodhart, 1974; Gordon, 1974; Nakano \& Muramatsu, 1976).

The response of the RES to stress, endocrine substances, and endotoxins has also been investigated by a number of researchers (Old, Clark, Benacerraf, \& Goldsmith, 1960; Park \& Scarborough, 1972; Stowe,
1977). Alterations in RES activity during stress were demonstrated to correlate with changes in endocrine activity. For example, adrenalectomy or hypophysectomy have been shown to decrease RES activity in response to electric shock (Bilder, 1976; Keefe, Helman, \& Smith, 1967; Zweifach, 1960).

Unlike other aspects of the immune response, investigations regarding the role of hypothalamic mechanisms on RES activity have been largely ignored (Segal, Izak, \& Feldman, 1971; Shekoyan, Khasman, \& Uchitel, 1975). Because hypothalamic mechanisms have been shown to interact with immune responses involving antibody synthesis or hypersensitivity, the possibility of hypothalamic involvement in RES activity was assessed in the present experiment.

\section{METHOD}

The subjects were eight experimentally naive male albino $250-\mathrm{g}$ Sprague-Dawley rats obtained from ARS Sprague-Dawley, Madison, Wisconsin. Each animal was individually housed, allowed food and water ad lib throughout the experiment, and handled daily for a minimum of 5 min.

Animals were first implanted with polyethylene cannulas in the right jugular vein according to the method outlined by Weeks (Note 1). Following recovery from the cannulation procedure, the animals were placed in a Kopf stereotaxic apparatus (Kopf Instruments, Tujunga, California) and electrodes were implanted bilaterally in the ventromedial area of the hypothalamus. The coordinates used for implantation were: $6.7 \mathrm{~mm}$ anterior of the interaural line, $.75 \mathrm{~mm}$ lateral of the midsaggital sinus, and $9.5 \mathrm{~mm}$ ventral from the dural surface of the brain (Sherwood \& Timiras, 1970). Sodium pentobarbital anesthesia $(50 \mathrm{mg} / \mathrm{kg})$ was used in all surgical procedures. 
Following the return of subjects to their preoperative weights (7-10 days), the tail of each animal was clipped and a $.025-\mathrm{ml}$ blood sample was taken between 10:00 a.m. and 12:00 noon. This blood sample, which followed electrode implantation, served as a baseline measure. After the baseline sample was taken, a suspension of carbon particles (Koor-I-Noor Industries, Bloomsfield, New Jersey) mixed with a $9 \%$ physiological saline, resulting in a concentration of $25 \mathrm{mg} / \mathrm{ml}$, was injected through the jugular cannula of each subject at a ratio of $5 \mathrm{mg} / \mathrm{kg}$. Blood samples were then taken from the tail vein every minute for $15 \mathrm{~min}$ and lysed in a 4-ml $.1 \%$ solution of sodium carbonate $\left(\mathrm{NA}_{2} \mathrm{CO}_{3}\right)$. During the tail-clipping procedure, subjects were not anesthetized and were allowed to rest in the crook of the experimenter's arm. Since the subjects were well habituated to handling, there was little resistance to this procedure. All samples were subjected to spectrophotometric analysis by a Bausch and Lomb spectrophotometer (Bausch and Lomb, Rochester, New York) at a setting of $675 \mathrm{~nm}$, and each blood sample was read against a blank of $4 \mathrm{ml} .1 \% \mathrm{NA}_{2} \mathrm{CO}_{3}$. Optical densities were calculated by subtracting the absorbance value of the baseline blood sample from each of the 151 -min samples taken after the injection of the carbon suspension. The logarithms of these optical densities were calculated for each animal, and half-time clearance rates were determined by the formula $T 1 / 2=.301 / b$, where .301 is the logarithm of 2 and $b$ is the slope of the regression line based on a least squares regression equation utilizing the logarithms of the optical densities across time (Cantrell, 1972; Halpern, Bencerraf, \& Biozzi, 1953; Normann, 1973; Stowe, 1977).

Five days following the determination of carbon clearance rates for each animal, the subjects were placed in a standard Lehigh Valley operant chamber (Lehigh Valley, Beltsville, Maryland) and brain stimulation was delivered through a bifurcated cable by a Grass S48 brain stimulator (Grass Instruments, Quincy, Massachusetts). Each animal received one train of rectangular pulses (100 pps) every $5 \mathrm{sec}$ with the duration of each train lasting $1 \mathrm{sec}$. The intensity of brain stimulation was adjusted for each animal by beginning the first session at $100 \mu \mathrm{A}$ and moving up in $100-\mu \mathrm{A}$ increments until either a maximum of $600 \mu \mathrm{A}$ was reached or until vocalization or exaggerated motor behavior was observed. If an aversive reaction was noted, the intensity was reduced by $100 \mu \mathrm{A}$ increments until aversive effects were no longer observed and the resulting intensity was held constant for each animal throughout the experiment. After $2 \mathrm{~h}$ of brain stimulation, each animal was returned to his home cage for 4 days; at the end of this period, each was subjected to another carbon clearance test. After 4 days, each animal received brain stimulation, using the parameters outlined above, and RES activity was measured using the carbon clearance test $3,6,12$, or $24 \mathrm{~h}$ postbrain stimulation. The stimulation and carbon clearance measurement was repeated for all animals until RES activity was assessed at each poststimulation period for each animal. The sequence of postbrain stimulation measurement intervals $(3,6,12$, and $24 \mathrm{~h}$ ) was counterbalanced across subjects so that each interval served as the first, second, third, and fourth measurement for an equal number of animals. A 4-day period was imposed between each measurement to allow for the possibility of carryover effects.

At the conclusion of the study, all subjects were sacrificed by sodium pentobarbital anesthesia and perfused with a $10 \%$ Formalin solution. The brains were removed and embedded in celloidon. The tissue was sectioned at $30-\mu$ thickness and stained with thionin. Gross morphological examination of the liver, spleen, and kidneys was also performed.

\section{RESULTS}

The results presented in Figure 1 indicate that ventromedial hypothalamic stimulation decreases the

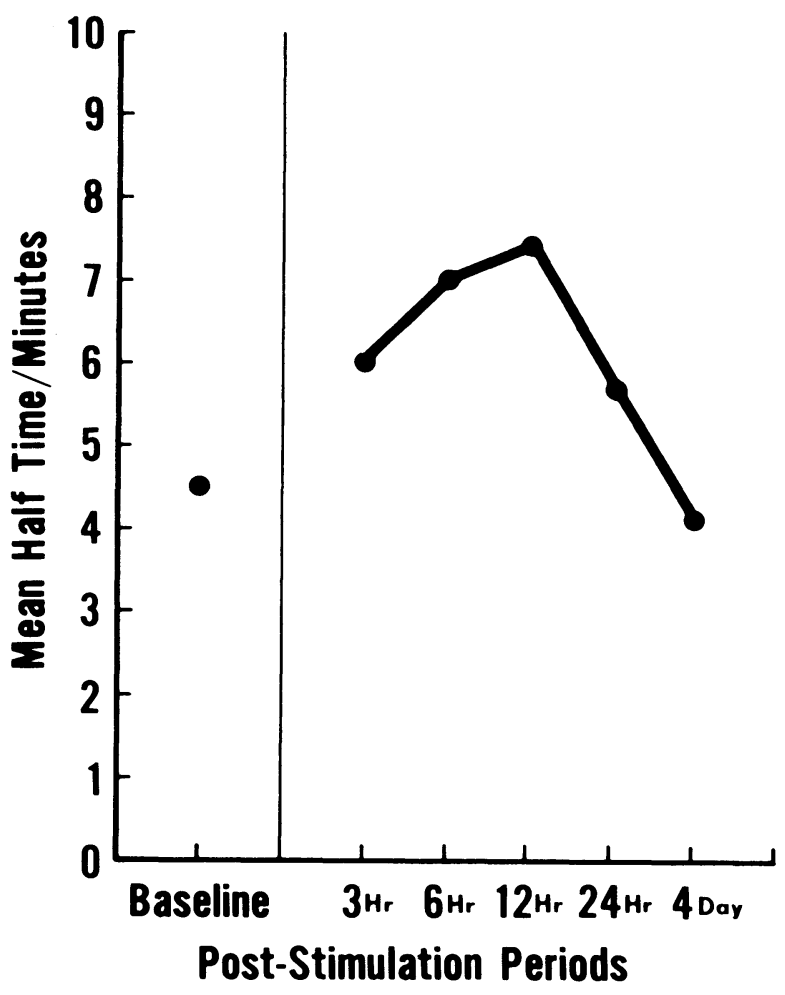

Figure 1. Mean half-time carbon clearance rate as a function of time since hypothalamic stimulation.

rate of carbon clearance from the bloodstream. Points along the abscissa represent the poststimulation from the average half-time carbon clearance rate at baseline. There was a strong correspondence between individual curves and the group curve shown in Figure 1. In fact, four of the eight individual curves were almost identical to the group curve, and six of the eight individual curves indicated greater half-time clearance rates for the 3-, 6-, 12-, and 24-h measurements than for the baseline or 4-day measurements. Also, no apparent relationship was observed between magnitude of effect and level of stimulation, although slight variations in electrode placement might cause different effects for equal levels of current. A one-way repeated ANOVA of difference scores indicates a significant overall effect $[F(4,28)=$ $5.47, \mathrm{p}<.01$ ]. By Newman-Keuls post hoc analysis of the difference scores, the 3-, 6-, 12-, and 24-h poststimulation periods do not differ from each other but show a significant increase from the 4-day measure. However, a subsequent trend analysis to determine the shape of the function across the 3-, 6-, 12-, and 24-h measurement periods indicated a statistically significant quadratic trend $[F(1,28)=11.42$, $\mathrm{p}<.01$ ].

Histological results indicated that electrodes were in the ventromedial nucleus with no indication of le- 
sioning at the tip. Morphological examination showed the presence of carbon residues in the liver and spleen, but no indication of carbon uptake by the kidneys.

\section{DISCUSSION}

The results of this investigation suggest that central nervous system activity may have an effect on the RES by depressing macrophage function. In this study, carbon clearance rate is depressed, beginning at $3 \mathrm{~h}$ and reaching a peak at $12 \mathrm{~h}$; by the fourth day, the rate returns to baseline. Therefore, it appears that a 4-day interval is sufficient to eliminate carry-over effects. This period of time for recovery of RES to baseline following activation is comparable to that observed in previous research (Stowe, 1977). Because each measurement sequence was counterbalanced across subjects, cumulative effects of repeating the carbon clearance measurement procedure are controlled and thus could not produce systematic differences among the 3-, 6-, 12-, and 24-h measurements.

Because of the nonphysiological amount of stimulation, the pattern of results observed here is not necessarily indicative of changes following central nervous system activation in the intact animal. The effects observed in this study may be a result of stimulating pathways which produce endocrine secretion, autonomic changes, or direct immunosuppression by unknown mechanisms.

Some alternative explanations to the above interpretations include blood-pressure changes, vascular changes, prolonged hypothalamic afterdischarge, and general arousal produced by the experimental procedure; however, the time course of the present results does not appear to be consistent with these explanations. None of these alternative effects should persist for $24 \mathrm{~h}$, and none would be expected to increase for as long as $12 \mathrm{~h}$ poststimulus. Also, the carbon clearance technique is not simply a measure of liver blood flow (Normann, 1973), and this is important, because the liver is the primary organ responsible for the removal of intravenously injected colloids. Diurnal variation in RES activity is an alternative explanation which does appear to be consistent with the time course observed because the 12 -h measurement shows the greatest decrease in carbon clearance rates. However, the 4-day measure is different statistically from the 3- and 24-h measurements, but these three measurements were taken at approximately the same time of day. Also, the 6- and 12-h measures were very close in clearance rates, but the 6-h measurement was taken in the early afternoon, while the 12-h measurement was taken in the evening.

Although the alternative explanations mentioned above cannot be completely ruled out, they do not seem to adequately explain these findings. While suppression of RES activity observed in the present study implicated the ventromedial area, research previously cited in the introduction section demonstrated effects of lesions or stimulation in various areas of the hypothalamus on immune measures which correlate highly with RES activity. Therefore, it is likely that stimulating other areas of the hypothalamus would influence RES activity. Thus, the present results suggest that RES activity may be under central nervous system modulation via as yet undefined hypothalamic mechanisms. The results also indicate that these pathways can decrease phagocytic activity in the reticuloendothelial system which operated in conjunction with other parts of the immune system to provide resistance against invading organisms. The precise way in which reticuloendothelial system activity interacts with antibody production is unknown, yet, based on the present results and previous studies on antibody formation, both are influenced by hypothalamic mechanisms. Therefore, research on the relationship between the immune system and the hypothalamus should assess changes in both antibody formation and reticuloendothelial system activity concurrently.

The far-reaching implications of these data have potential importance for our understanding of the functioning of immune systems in the intact organism in its environment. The hypothalamus participates in a variety of functions important to survival and the maintenance of homeostasis. For example, the hypothalamus is known to control many aspects of metabolism (Bray \& York, 1979; EmmettOglesby, Lacko, Harrell, \& Jordan, 1979; Harrell \& Remley, 1973), and Powley (1977), in a comprehensive review, summarizes evidence that this control is modified by conditioning. The possibility exists that hypothalamic control of the immune system is also altered by conditioning. The demonstration that conditioning of the immune system occurs using a Pavlovian classical procedure is consistent with this speculation (Ader \& Cohen, 1975; Rogers, Reich, Strom, \& Carpenter, 1976). Specifically, these studies demonstrate that rats receiving pairings of saccharine and an immunosuppressive agent, cyclophosphamide, show a decrease in antibody titers when presented with saccharine alone. This demonstration strengthens the possibility that the immune system is modifiable through environmental conditioning.

In conclusion, research on hypothalamic involvement in immune processes, metabolic adjustments, and concomitant changes in motivational state suggests a neural mechanism which alters immunoresponsivity. The possibility also exists that this neural mechanism is modified by conditioning. The direction of change may be either suppression, as demonstrated in the present study, or activation (Besedovsky 
\& Sorkin, 1977). Further studies exploring the effects of stimulating different hypothalamic areas while measuring both RES activity and antibody formation should help to clarify the exact nature of the neural influence. This may lead to a better understanding of the complex relationships observed in the realm of psychosomatic illness.

\section{REFERENCE NOTE}

1. Weeks, J. R. Cardiovascular techniques using unanesthetized and freely moving rats. Unpublished manuscript, Upjohn Co., Kalamazoo, Michigan, 1967.

\section{REFERENCES}

Ader, R., \& Cohen, N. Behaviorally conditioned immunosuppression. Psychosomatic Medicine, 1975, 37, 333-340.

Besedovsky, H., \& Sorkin, E. Network of immune-endocrine interactions. Journal of Clinical Experimental Immunology, 1977, 27, 1-12.

BILder, G. E. Activity of the reticuloendothelial system followin endocrine manipulation in rats. Journal of the Reticuloendothelial Society, 1976, 19, 11-17.

BrAY, G., \& York, D. Hypothalamic and genetic obesity in experimental animals: An autonomic and endocrine hypothesis. Physiological Reviews, 1979, 59, 719-784.

Cantrell, E. T. Induction of benzpyrene hydroxylase in parenchymal and Kupfer cells of rat liver. Unpublished doctoral dissertation, Baylor University, 1972.

CARR, I. The macrophage: $A$ review of ultrastructure and function. New York: Academic Press, 1973.

Emmett-Oglesby, M. W., Lacko, A. G., Harkell, E. H., \& JoRdAN, J. Cholesterol metabolism in the rat following lesions of the diencephalon. Artery, 1979, 5, 474-485.

Fessel, W. J., \& ForsyTh, R. Hypothalamic role in control of gamma globulin levels. Archives of Allergy, 1963, 771. (Abstract)

Friedman, H. H. Reticuloendothelial system and passive transfer of endotoxin tolerance. Annals of the New York Academy of Sciences, 1960, 88, 99-106.

Frobisher, M., Hindsill, R., Crabtree, K., \& Goodhart, C. Fundamentals of microbiology. Philadelphia: Saunders, 1974.

Gordon, B. Essentials of immunology. Philadelphia: Davis, 1974.

Halpern, B. N., Benacerraf, B., \& Biozzi, G. Quantitative study of the granulospecific activity of the reticuloendothelial system. I. The effect of the ingredients present in india ink and of substances affecting blood clotting in vivo. British Journal of Experimental Pathology, 1953, 34, 426-440.

HARRELL, E. H., \& REMLEY, N. R. The immediate development of behavioral and biochemical changes following ventromedial hypothalamic lesions in rats. Behavioral Biology, 1973, 9, 43-46.

Kavetsky, R., Turkevich, N., Akimova, R., Khayetsky, I., \& Matuechuck, Y. Induced carcinogenesis under various influences on the hypothalamus. Annals of the New York Academy of Sciences, 1969, 164, 517-519.
Keefe, B., Helman, S., \& SMith, J. J. RES response to hypophysectomy in the rat. Journal of the Reticuloendothelial Society, $1967,4,177-189$.

KoRneva, F., \& KHAI, L. Effect of destruction of hypothalamic areas on immunogenesis. Fiziologicheskii Sechenov, 1963, 49, 42.

Macris, N. T., Schiavi, R., Camerino, M., \& Stein, M. Effect of hypothalamic lesion on the immune process in the guinea pig. American Journal of Physiology, 1975, 19, 1205-1209.

Nakano, K., \& Muramatsu, S. Studies of the role of macrophages in the antibody response of mice: Stimulation of T-cell dependent antibody responses by tolerogenic soluble antigen trapped by macrophages. Journal of the Reticuloendothelial Society, 1976, 19, 347-359.

Normann, S. J. Reticuloendothelial system function. VI. Experimental alterations influencing the correlation between portal blood flow and colloid clearance. Journal of the Reticuloendothelial Society, 1973, 13, 47-60.

Old, L., Clarke, D., Benacerraf, A., \& Goldsmith, M. The reticuloendothelial system and the neoplastic process. Annals of the New York Academy of Sciences, 1960, 88, 264-280.

Park, L., \& Scarborough, B. Reticuloendothelial stimulation and depression: Effects on conditioned suppression. Journal of the Reticuloendothelial Society, 1972, 12, 629-639.

Powley, T. L. The ventromedial hypothalamic syndrome, satiety and a cephalic phase hypothesis. Psychological Review, 1977, 84, 89-126.

Rogers, M. P., Reich, P., Strom, T. B., \& Carpenter, C. B. Behaviorally conditioned immunosuppression: Replication of a recent study. Psychosomatic Medicine, 1976, 38, 447-451.

Schinvi, R., Adams, J., \& Stein, M. Effect of hypothalamic lesions on histamine toxicity in the guinea pig. American Journal of Physiology, 1966, 211, 1269-1273.

Segal, R., Izak, G., \& Feldman, S. Augmented red cell sequestration after prolonged electrical stimulation of the posterior hypothalamus in rats. Journal of the Reticuloendothelial Society, $1971,9,225-236$.

Sheroyan, V., Khasman, E., \& Uchitel, I. The effect of structures of the anterior and posterior hypothalamus on the engulfment and digestion of the antigen by macrophages and the India ink clearance. Journal of Microbiology and Epidemiology, 1975, 10, 131-135.

Sherwood, N. M., \& Timiras, P. S. A stereotaxic atlas of the developing rat brain. Berkeley: University of California Press, 1970.

STowe, J. E. The effects of stimulation and depression of the reticuloendothelial system on Sidman avoidance behavior (Doctoral dissertation, North Texas State University, 1977). Dissertation Abstracts International, 1977, 38, 1460. (University Microfilms No. 77-19685)

ZWEIFACH, B. W. The contribution of the reticuloendothelial system to the development of tolerance to experimental shock. Annals of the New York Academy of Sciences, 1960, 88, 203-212.

(Received for publication November 17, 1980; revision accepted March 23, 1981.) 